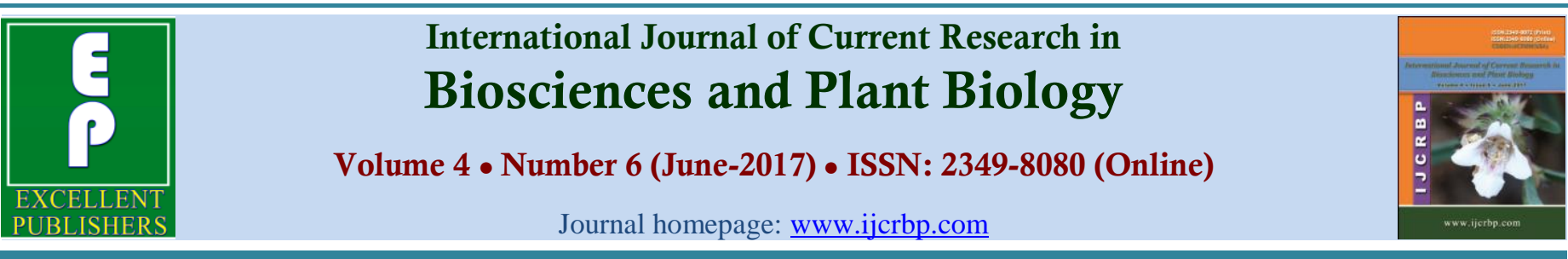

\title{
Diversity of Wild Edible Plants in Dhadgaon Block of Nandurbar District in Maharashtra, India
}

\author{
Vitthal Kauthale*, Dilip Kulkarni, Lilesh Chavan, Sanjay Patil and Anjali Nalawade
}

BAIF, Development Research Foundation, Central Research Station Urulikanchan, Tal. - Haveli, Dist. - Pune, 412 202, Maharashtra, India

*Corresponding author.

\begin{tabular}{|c|c|}
\hline Abstract & Article Info \\
\hline $\begin{array}{l}\text { Present study deals with survey and documentation of wild edible plants (WEPs) in } \\
\text { Dhadgaon/Akrani region of Nandurbar district. Present paper is the first documentation of } \\
\text { WEPs used by local communities in Nandurbar district. Data on about } 91 \text { wild edible } \\
\text { plant species in } 69 \text { genera of } 40 \text { families was recorded. Majority of WEPs belongs to } \\
\text { family Fabaceae (11 species), Araceae (7), Asparagaceae (6), Malvaceae (6), } \\
\text { Amaranthaceae (5), Apocynaceae (5), Asteraceae (4), Dioscoriaceae (4), Rubiaceae (4) } \\
\text { and Cucurbitaceae (3). The genera represented by the highest number of species are } \\
\text { Chlorophytum (5 species) followed by Dioscorea (4), Amaranthus (3) and } 2 \text { species each } \\
\text { from Amorphophallus, Arisaema, Bauhinia, Cassia, Launea, Moringa, Smithia, Sterculia, } \\
\text { Vigna and Ziziphus. Most of the species were consumed as leafy vegetable (46 species) or } \\
\text { fruits ( } 34 \text { species). More than one plant part is used from } 14 \% \text { of the species. These wild } \\
\text { edible plants are sources of food security for local people, especially those living in } \\
\text { remote rural areas. Some species are wild relatives of crops that could be used for crop } \\
\text { improvement program in future. Several species have potential values for further } \\
\text { domestication and commercial exploitation which will help in biodiversity conservation } \\
\text { and sustainable utility. }\end{array}$ & $\begin{array}{l}\text { Keywords } \\
\text { Food security } \\
\text { Nandurbar } \\
\text { Nutrition } \\
\text { Wild edibles }\end{array}$ \\
\hline
\end{tabular}

\section{Introduction}

More than 12,000 plant species considered edible by humans, i.e., plants for human consumption account for about $5 \%$ of the total plant species of the world (Kunkel, 1983). It is estimated that in India about 800 species are consumed as wild edible plants (Singh and Arora, 1978). Wild edible plants (WEPs) refer to species that are harvested or collected from their wild natural habitats and used as food for human consumption (Lulekal et al., 2011; Heywood, 2011;
Seal, 2012). WEPs play a major role in meeting the nutritional requirement of the tribal population in remote areas (Sundriyal and Sundriyal, 2001). WEPs serve as supplementary food for non-indigenous people and are one of the primary sources of cash income for poor communities (Uprety et al., 2012; Ghorbani et al., 2012; Menendez-Baceta et al., 2012). WEPs have an important role in ensuring food security and improve the nutrition in the diets of many people in developing countries (Lulekal et al., 2011; Ghorbani et al., 2012). WEPs are potential sources of species for 
domestication and provide valuable genetic traits for developing new crops through breeding and selection (Pandey et al., 2008; Ford-Lloyd et al., 2011).

India has a tribal population of 42 million, of which some 60 per cent live in forest areas and depend on forests for various edible products (Jana and Chauhan, 1998). Several researchers have documented the WEPs used in the diet by tribes in various parts of India, nutritive values of WEPs and need for revival of knowledge associated with WEPs (Sundriyal and Sundriyal, 2004; Konsam et al., 2016; Mahapatra and Panda, 2012; Khyade et al., 2009).

Nandurbar district is a tribal district situated in the North West of Maharashtra. The tribal population consisting of Bhils, Gamits, Koknas, Pawara forms about $40 \%$ of the population of the district (Patil, 2010). These tribal communities are mainly dependent on forest products. They still use several wild plants growing around them for different purposes like food, fodder, medicine, timber, firewood, etc.

Nandurbar and adjoining areas are well studied in ethnobotany point of view. The notable works have been published by Tayade et al. (2016), Patil and Bhaskar (2005), Padavi and Patil (2013), Lande and Kalase (2014), Patil (2003), and Quazi and Molvi (2014); however these works focus on medicinal properties of plants. Very few records were made on wild edible plants. During working on Maharashtra Gene Bank Programme, it was found that Dhadgaon region is very rich in wild food diversity; and in literature the data on WEPs is very limited in Dhadgaon region of Nandurbar district. Present paper reveals the information on exploration and documentation of WEPs with respect to correct identification, habit and habitat, edible part, processing method, season of availability, domestication and conservation.

\section{Materials and methods}

\section{Study area}

The study area is situated in Satpuda ranges. The study was carried out in 12 villages in Dhadgaon region of Nandurbar district. Nandurbar district is situated in Northern part of the state of Maharashtra bordering the state of Gujarat and Madhya Pradesh. Nandurbar district is situated between $20^{\circ}$ to $21^{\circ}$ North latitude and $74^{\circ} 55^{\prime}$ and $76^{\circ} 59^{\prime}$ East longitude. The forest in the district covers an area of 4732.199 sq. km. (Tayade et al., 2016) (Fig. 1).

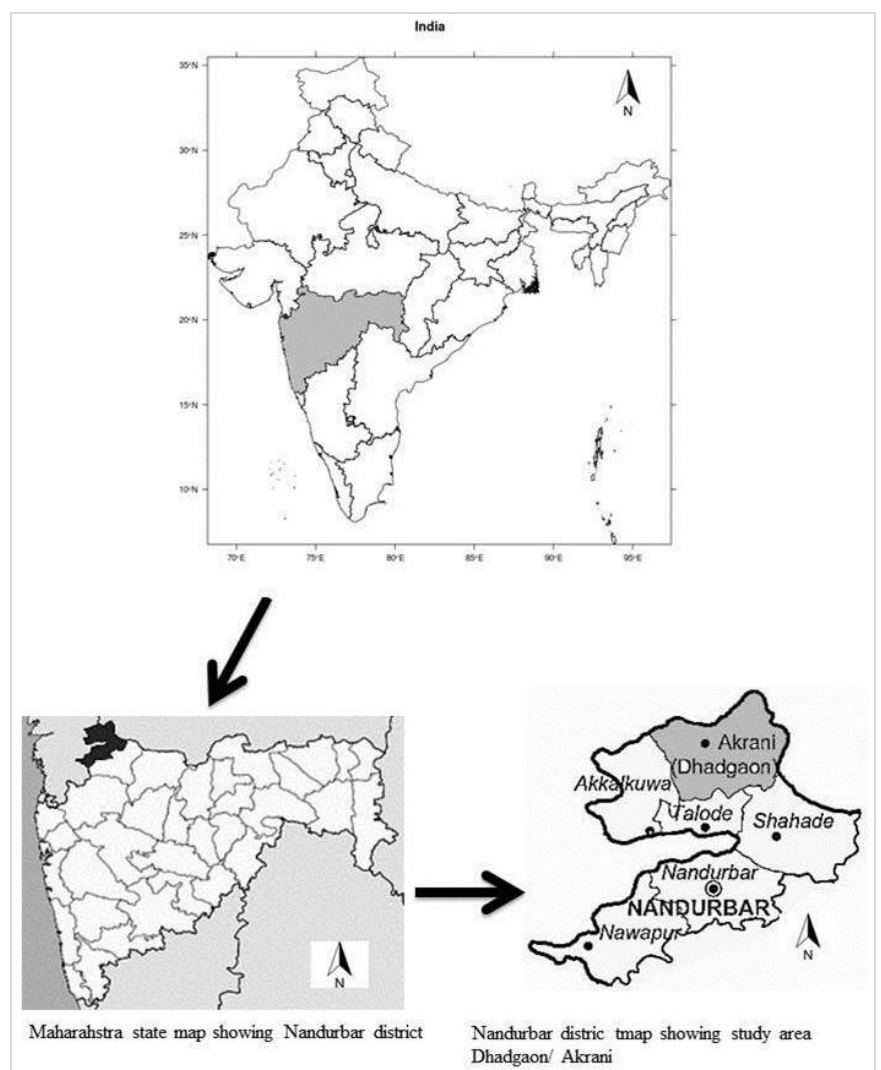

Fig. 1: Location of the area covered in an investigation into the wild edible plants in Dhadgaon (Akrani).

\section{Data collection}

For data collection household survey and field studies were carried out during different seasons of the year (2014-2016). Data of wild edible plants were documented through different interview methods [participatory rural appraisal (PRA), direct observation, semi-structured interviews, key informant interviews, individual discussions, focus group discussions and questionnaires]. Besides these, exhibition cum recipe competition of wild edible plants was organized for indepth understanding of plant diversity.

During survey, the local name, parts used, habit and habitat, collection period and preparation methods, propagation, utility other than food, and people's perceptions about plants were recorded and few specimens were collected. Collected plant specimens were identified by using relevant scientific literature and regional/ state flora (Cooke, 1967; Sharma et al., 1996; Singh and Karthikeyan, 2000; Singh et al. 2001). 


\section{Results and discussion}

\section{Diversity of species, growth forms and edible parts}

During present study 91 species of wild edible plants were documented that belongs to 69 genera 40 families of flowering plants (Annexure 1). Majority of edible plants belongs to family Fabaceae (11 species), Araceae (7), Asparagaceae (6), Malvaceae (6), Amaranthaceae (5), Apocynaceae (5), Asteraceae (4), Dioscoriaceae (4), Rubiaceae (4), Cucurbitaceae (3). The genera represented by the highest number of species are Chlorophytum (5 species) followed by Dioscorea (4),
Amaranthus (3) and 2 species each from Amorphophallus, Arisaema, Bauhinia, Cassia, Launea, Moringa, Smithia, Sterculia, Vigna and Ziziphus (Fig. 2). Documented flowering plants distributed into different habits with herbs (42\%), trees (37\%), Climber (13\%) and shrubs (8\%) (Fig. 3). Out of these recorded wild edible plants $41 \%$ species has edible leaves/ tender stem, $29 \%$ species has edible fruits, $16 \%$ species has edible inflorescence and flowers, $9 \%$ species has storage parts (Tuber, Rhizome, Corm, bulbils etc.) and $5 \%$ species has edible seeds. The most frequently used parts are young leaves/ stem, fruits and flower/ inflorescence (Fig. 4).

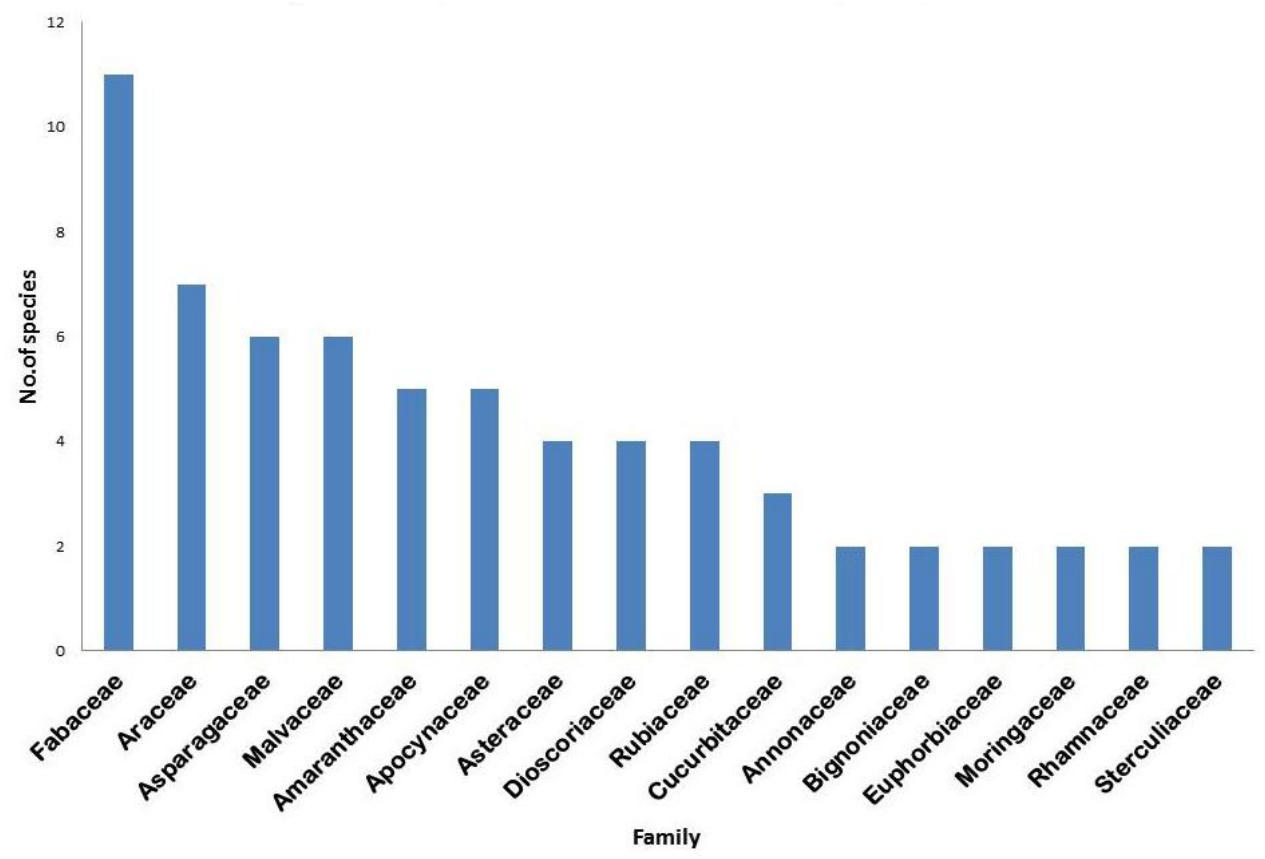

Fig. 2: Family-wise distribution of wild edible plant species.

More than one plant part is used from about $14 \%$ of the species e.g. petioles and leaves of Colocasia esculenta used for preparation of various dishes of vegetable as well as corms are also edible and stored for lean period; leaves, flower and tender fruits of Bauhinia racemosa are edibles; tender leaves, young flowers, fruits and seeds of Tamarindus indica are edible.

\section{Storage of edible plant parts}

Underground tubers and bulbils of plants like Amorphophallus sp., Colocasia esculenta, Dioscorea, are often dried in the sun after collection and stored. Leaves of various plants like Amaranthus sp. Sauromatum venosum, Hibiscus sp. and calyx of Hibiscus sp. are also stored by sun drying for lean period. Pickles are made of fruits like Capparis zeylanica, Carissa carandas, Carrisa congesta, Catunaregam spinosa, Garuga pinnata, Meyna laxiflora, Radermachera xylocarpa, Spondias pinnata, and Tamilnadia uliginosa, etc.

\section{Multiple uses of wild edible plants}

Many wild edible plants have uses other than food, e.g. Agave sp. is used as hedge, fiber plant and as soil binder.

Bambusa arundinacea is important timber plant and many household goods are made from it. Cassia fistula, Celosia argentea, Chlorophytum borivilianum Holarrhena pubescens and Wrightia tinctoria are used as medicine. Catunaregam spinosa is used in grain storage; Crotalaria juncea is grown for green manure; 
Hibiscus sp. and Bombax ceiba are fiber plant; Alocasia macrorhizos, Basella alba, Bauhinia sp., Dioscorea alata, and Thespesia populnea are grown as ornamental or avenue plants.

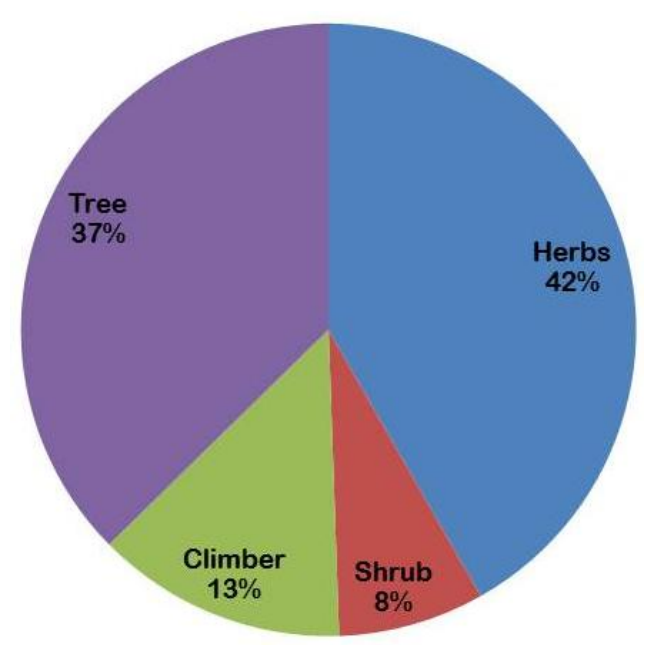

Fig. 3: Diversity in the habits of wild edible plants.

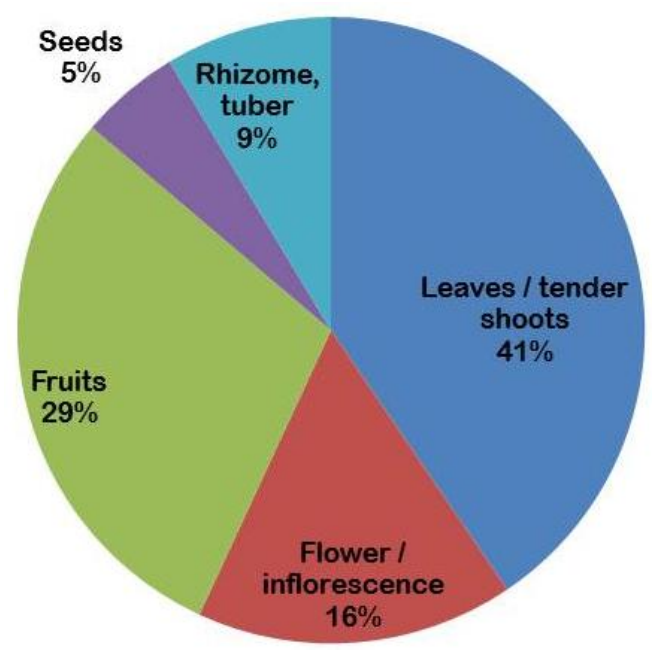

Fig. 4: Wild edible plants and their edible parts used.

\section{Role of wild relatives of crop for genetic improvement and crop production}

Wild crop relatives are of great importance to maintain the productivity and stability of traditional agroecosystems (Meilleur and Hodgkin 2004; Harlan, 1965). Some of the wild relatives of fruit, vegetable and fiber crops documented in this study are Amaranthus sp., Amorphophallus commutatus, Ensete superbum, Hibiscus sp., Momordica dioica, Moringa concanensis, Vigna sp., Ziziphus rugosa, etc. Conservation through domestication of these plant species will help in crop genetic improvement program in future.

\section{Domestication and wider use of wild edible plants}

All domesticated plants had passed during the course of human civilization, as the entire spectrum represents a living analogue of the wild to semi-wild to domesticated continuum (Asfaw and Tadesse, 2001). Periodic use, occasional purposeful planting and harvesting, marketability, and proximal growth with gardens, crop fields, and living quarters disclose the position of wild edible species in the line-up of useful plants (Asfaw and Tadesse, 2001). During present study many edible plant species like Amorphophallus sp., Colocasia esculenta, Dioscorea sp., Emblica officinalis, Hibiscus sp., Tamarindus indica and Vigna mungo occurred in wild as well as cultivated forms while some plants like Agave sp., Bauhinia purpurea, Moringa oleifera, Sesbania grandiflora, Alocasia macrorhizos, etc. are introduced in study area for various purposes; however in course of time they get naturalized and people are utilizing them commonly.

The fruits of Annona squamosa, Carissa carandas, Carrisa congesta, Garuga pinnata, Meyna laxiflora are widely sold on roadsides and in the market place for income generation. Although Annona squamosa is fruit crop in other regions of India, but in study area it occurs in nature only, and there is great scope for domestication of such wild edible plants in study area. Fruits of many species can be considered for value addition and processing into more palatable and storable food products e.g. Annona sqaumosa, Tamarindus indica, Capparis zeylanica, Carissa carandas, Carrisa congesta, Catunaregam spinosa, Garuga pinnata, Meyna laxiflora, Radermachera xylocarpa, Spondias pinnata, and Tamilnadia uliginosa can be processed into jam, jelly, candy, pickles, sharbat (juices), ice-creams, various types of sweets etc. The domestication of potential wild edible plants along integration into existing land use will increase livelihoods of rural community. With better market linkages in place, wild edibles could also be promoted for use by urban dwellers.

\section{Conclusion}

Present paper is the first documentation of WEPs used by local communities in Nandurbar district. Local community not only cultivates various crops, but also collects wild edible plants as food. Present study showed the diversity of WEPs and related indigenous knowledge in this area. WEPs provide food and nutrients, such as 
essential amino acids, various vitamins and minerals which are needed to keep healthy and enhance immunity against diseases and infections to local communities. If properly harvested, WEPs could be the source of cash income for local people.

Present study may provide basic information for priority list preparation for domestication, conservation, possibly further exploitation, and will preserve local traditional knowledge.

\section{Conflict of interest statement}

Authors declare that they have no conflict of interest.

\section{Acknowledgement}

Authors are thankful to Rajiv Gandhi Science and Technology Commission, Govt. of Maharashtra, for financial support.

\section{References}

Asfaw, Z., Tadesse, M., 2001. Prospects for Sustainable Use and Development of Wild Food Plants in Ethiopia. Econ. Bot. 55(1), 47-62.

Cooke, T., 1967 (Rpr.). The Flora of the Presidency of Bombay. Vol. I, II, III. Botanical Survey of India. Culcutta.

Ford-Lloyd, B. V., Schmidt, M., Armstrong, S. J., Barazani, O., Engels, J., Ge, S., Hadas, R., Hammer, K., Kell, S. P., Kang, D., K. Khoshbakht, Li, Y., Long, C. L., Lu, B. R., Ma, K. P., Nguyen, V. T., Qiu, L. J., Wei, W., Zhang, Z. W., Maxted, N., 2011. Crop wild relatives undervalued, underutilized and under threat? BioScience. 61, 559-565.

Ghorbani, A., Langenberger, G., Sauerborn, J., 2012. A comparison of the wild food plant use knowledge of ethnic minorities in Naban River Watershed National Nature Reserve, Yunnan, SW China. J. Ethnobiol. Ethnomed. 8, 17.

Harlan, J. R., 1965. The possible role of weed races in the evolution of cultivated plants. Euphytica. 14, 173-176.

Heywood, V. H., 2011. Ethnopharmacology, food production, nutrition and biodiversity conservation: Towards a sustainable future for indigenous peoples. J. Ethnopharmacol. 137, 1-15.

Jana, S. K., Chauhan, A. S., 1998. Wild edible plant of Sikkim Himalaya. J. Non-Timb. For. Prod. 5(1 \& 2), 20-28.
Khyade, M. S., Kolhe, S. R., Deshmukh, B. S., 2009. Wild edible plants used by the tribes of Akole Tehsil of Ahmednagar District (MS), India. Ethnobot. Leaflets. 13, 1328-1336.

Konsam, S., Thongam, B., Handique, A. K., 2016. Erratum to: Assessment of wild leafy vegetables traditionally consumed by the ethnic communities of Manipur, Northeast India. J. Ethnobiol. Ethnomed. 12,9 .

Kunkel, G. 1983. Plants for Human Consumption. Koeltz Scientific Books, Germany.

Lande, L. J., Kalase, A. T., 2014. Indigenous herbal medicines used by tribal people in Satpuda Mountain. Int. Science J. 1(1), 65-69.

Lulekal, E., Asfaw, Z., Kelbess, E., Van Damme, P., 2011. Wild edible plants in Ethiopia: A review on their potential to combat food insecurity. Africa Focus. 24, 71-121.

Mahapatra, A. K, Panda, P. C., 2012. Wild edible fruit diversity and its significance in the livelihood of indigenous tribals: Evidence from eastern India. Food Sec. 4(2), 219-234.

Meilleur, B. A., Hodgkin, T., 2004. In-situ conservation of crop wild relatives: Status and trends. Biodivers. Conserv. 13, 663-684.

Menendez-Baceta, G., Aceituno-Mata, L., Tardío, J., Reyes-García, V., Pardo-de-Santayana, M., 2012. Wild edible plants traditionally gathered in Gorbeialdea (Biscay, Basque Country). Genet. Resour. Crop Evol. 59, 1329-1347.

Padavi, P. C., Patil, D. A., 2013. Ethnobotanical and philological investigations in Akkalkuwa Tehsil of Nandurbar District (Maharashtra, India). Sch. Acad. J. Biosci. 1(6), 267-276.

Pandey, A., Tomer, A. K., Bhandari, D. C., Pareek, S. K., 2008. Towards collection of wild relatives of crop plants in India. Genet. Resour. Crop Evol. 55, 187-202.

Patil, D. A., 2010. Elements of ethnotaxonomy in Dhule and Nandurbar Districts (Maharashtra). J. Ecobiotechnol. 2/3, 18-25.

Patil, H. M., Bhaskar, V. V., 2005. Medicinal uses of plants by tribal medicine men of Nandurbar District in Maharashtra. Nat. Prod. Rad. 5(2), 125-130.

Patil, S. H., 2003. Ethno-medico-zoological studies on Nandurbar district of Maharashtra. Indian J. Trad. Knowl. 2(3), 297-299.

Quazi, M. A., Molvi, K. I., 2014. Ethnomedicinal survey of Meyna laxiflora in tribes of Akkalkuwa, Nandurbar District. Int. J. Pharm. BioSci. 5(3), 225230 . 
Seal, T., 2012. Evaluation of nutritional potential of wild edible plants, traditionally used by the tribal people of Meghalaya state in India. Amer. J. Plant Nutr. Fertil. Tech. 2, 19-26.

Sharma, B. D., Karthikeyan, S., Singh, N. P., 1996. Flora of Maharashtra State, Monocotyledons. Botanical Survey of India. Calcutta.

Singh, H. B., Arora, R. K., 1978. Raishan (Digitaria sp.)-A minor millet of Khasi Hills, India. Econ. Bot. 26, 376-380.

Singh, N. P., Karthikeyan, S., 2000. Flora of Maharashtra State - Dicotyledons. Vol. I. Botanical Survey of India. Calcutta.

Singh, N. P., Lakshminarasimhan, P., Karthikeyan, S.,
Prasanna, P.V., 2001. Flora of Maharashtra State Dicotyledones. Vol. II. Botanical Survey of India, Calcutta.

Sundriyal, M., Sundriyal, R. C., 2001. Wild edible plants of the Sikkim Himalaya: Nutritive values of selected species. Econ. Bot. 55(3), 377-390.

Tayade, S. K., Pathak, S. S., Patil, V. K., 2016. Ethnobotanical study of some useful plants of Nandurbar District (Maharashtra). Int. J. Scient. Res. 5(1), 160-162.

Uprety, Y., Poudel, R., Shrestha, K., Rajbhandary, S., Tiwari, N., Shrestha, U., Asselin, H., 2012. Diversity of use and local knowledge of wild edible plant resources in Nepal. J. Ethnobiol. Ethnomed. 8, 16.

\section{How to cite this article:}

Kauthale, V., Kulkarni, D., Chavan, L., Patil, S., Nalawade, A., 2017. Diversity of wild edible plants in Dhadgaon Block of Nandurbar District in Maharashtra, India. Int. J. Curr. Res. Biosci. Plant Biol. 4(6), 62-73.

doi: https://doi.org/10.20546/ijcrbp.2017.406.007 
Annexure 1: List of WEPs with common name, scientific name, habit and habitat, edible parts used by local community in Nandurbar district.

\begin{tabular}{|c|c|c|c|c|c|c|c|}
\hline $\begin{array}{l}\text { Sl. } \\
\text { No. }\end{array}$ & $\begin{array}{l}\text { Common } \\
\text { name }\end{array}$ & Scientific name & Family & Habit & Habitat & Edible part & Season \\
\hline 1. & Ghaypat & $\begin{array}{l}\text { Agave americana } \\
\text { L. }\end{array}$ & Asparagaceae & Robust herbs & $\begin{array}{l}\text { Bunds of } \\
\text { social } \\
\text { forestry, hill } \\
\text { slopes }\end{array}$ & $\begin{array}{l}\text { Newly } \\
\text { emerged } \\
\text { shoots }\end{array}$ & Rainy \\
\hline 2. & Ghaypat & $\begin{array}{l}\text { Agave sisalana } \\
\text { Perrine ex } \\
\text { Engelm. }\end{array}$ & Asparagaceae & Robust herbs & $\begin{array}{l}\text { Bunds of } \\
\text { social } \\
\text { forestry, hill } \\
\text { slopes }\end{array}$ & $\begin{array}{l}\text { Newly } \\
\text { emerged } \\
\text { shoots }\end{array}$ & Rainy \\
\hline 3. & Kali alu & $\begin{array}{l}\text { Alocasia } \\
\text { macrorhizos G. } \\
\text { Don }\end{array}$ & Araceae & Herbs & $\begin{array}{l}\text { Marshy and } \\
\text { moist places }\end{array}$ & Leaves & Rainy \\
\hline 4. & $\begin{array}{l}\text { Dethachi } \\
\text { bhaji/ Dehgad }\end{array}$ & $\begin{array}{l}\text { Alternanthera } \\
\text { sessilis (L.) R. Br. } \\
\text { ex DC. }\end{array}$ & Amaranthaceae & Herbs & $\begin{array}{l}\text { Weed of } \\
\text { cultivated } \\
\text { fields and } \\
\text { waste lands }\end{array}$ & $\begin{array}{l}\text { Tender } \\
\text { stem and } \\
\text { leaves }\end{array}$ & Rainy \\
\hline 5. & $\begin{array}{l}\text { Tandalya/ } \\
\text { Tandulka }\end{array}$ & $\begin{array}{l}\text { Amaranthus } \\
\text { roxburghianus } \\
\text { H.W. Kung. }\end{array}$ & Amaranthaceae & Herbs & $\begin{array}{l}\text { Weed of } \\
\text { cultivated } \\
\text { fields and } \\
\text { waste lands }\end{array}$ & $\begin{array}{l}\text { Tender } \\
\text { stem and } \\
\text { leaves }\end{array}$ & Rainy \\
\hline 6. & Kate math & $\begin{array}{l}\text { Amaranthus } \\
\text { spinosus L. }\end{array}$ & Amaranthaceae & Herbs & $\begin{array}{l}\text { Weed of } \\
\text { cultivated } \\
\text { fields and } \\
\text { waste lands }\end{array}$ & $\begin{array}{l}\text { Tender } \\
\text { stem and } \\
\text { leaves }\end{array}$ & Rainy \\
\hline 7. & Veda math & $\begin{array}{l}\text { Amaranthus } \\
\text { viridis } \mathrm{L} \text {. }\end{array}$ & Amaranthaceae & Herbs & $\begin{array}{l}\text { Weed of } \\
\text { cultivate } \\
\text { fields }\end{array}$ & $\begin{array}{l}\text { Tender } \\
\text { stem and } \\
\text { leaves }\end{array}$ & Rainy \\
\hline 8. & Sheval & $\begin{array}{l}\text { Amorphophallus } \\
\text { commutatus } \\
\text { (Schott) Engl. }\end{array}$ & Araceae & Herbs & $\begin{array}{l}\text { Forest } \\
\text { understory }\end{array}$ & $\begin{array}{l}\text { Corm, } \\
\text { Tender } \\
\text { leaves }\end{array}$ & Rainy \\
\hline 9. & Suran & $\begin{array}{l}\text { Amorphophallus } \\
\text { paeoniifolius } \\
\text { (Dennst.) } \\
\text { Nicolson }\end{array}$ & Araceae & Herbs & $\begin{array}{l}\text { Forest } \\
\text { understory }\end{array}$ & Corm & Rainy \\
\hline 10. & Sitaphal & $\begin{array}{l}\text { Anona squamosa } \\
\text { L. }\end{array}$ & Annonaceae & Small trees & Hill slopes & Fruits & Winter \\
\hline 11. & Pivla dhotara & $\begin{array}{l}\text { Argemone } \\
\text { mexicana } \mathrm{L} \text {. }\end{array}$ & Papaveraceae & Herbs & $\begin{array}{l}\text { Weed of } \\
\text { cultivated } \\
\text { fields and } \\
\text { waste lands }\end{array}$ & $\begin{array}{l}\text { Flower } \\
\text { stalks }\end{array}$ & $\begin{array}{l}\text { Through } \\
\text { out year }\end{array}$ \\
\hline 12. & Badada & $\begin{array}{l}\text { Arisaema murrayi } \\
\text { Graham }\end{array}$ & Araceae & Herbs & $\begin{array}{l}\text { Forest } \\
\text { understory }\end{array}$ & Corm & Rainy \\
\hline 13. & $\begin{array}{l}\text { Diva/ } \\
\text { Nagphani }\end{array}$ & $\begin{array}{l}\text { Arisaema } \\
\text { tortuosum (Wall.) } \\
\text { Schott }\end{array}$ & Araceae & Herbs & $\begin{array}{l}\text { Forest } \\
\text { understory }\end{array}$ & Corm & Rainy \\
\hline 14. & $\begin{array}{l}\text { Bambu } \\
\text { /Vasate }\end{array}$ & $\begin{array}{l}\text { Bambusa } \\
\text { arundinacea } \\
\text { (Retz.) Willd. }\end{array}$ & Poaceae & Herbs & $\begin{array}{l}\text { Hill slopes, } \\
\text { river banks }\end{array}$ & $\begin{array}{l}\text { Tender } \\
\text { shoots }\end{array}$ & Rainy \\
\hline 15. & Bhaji vel & Basella alba L. & Basellaceae & Climbers & $\begin{array}{l}\text { On hedges of } \\
\text { kitchen } \\
\text { garden }\end{array}$ & $\begin{array}{l}\text { Tender } \\
\text { leaves }\end{array}$ & $\begin{array}{l}\text { Through } \\
\text { out year }\end{array}$ \\
\hline
\end{tabular}




\begin{tabular}{|c|c|c|c|c|c|c|c|}
\hline $\begin{array}{l}\text { Sl. } \\
\text { No. }\end{array}$ & $\begin{array}{l}\text { Common } \\
\text { name }\end{array}$ & Scientific name & Family & Habit & Habitat & Edible part & Season \\
\hline 16. & Kanchan & $\begin{array}{l}\text { Bauhinia } \\
\text { purpurea } \mathrm{L} .\end{array}$ & Fabaceae & Trees & $\begin{array}{l}\text { Social } \\
\text { forestry } \\
\text { cultivated } \\
\text { along } \\
\text { roadsides }\end{array}$ & $\begin{array}{l}\text { Tender } \\
\text { leaves }\end{array}$ & $\begin{array}{l}\text { Through } \\
\text { out year }\end{array}$ \\
\hline 17. & $\begin{array}{l}\text { Shida (Pod) / } \\
\text { Apta (tender } \\
\text { leaves)/ } \\
\text { Kohrula } \\
\text { (Flower, leaf) }\end{array}$ & $\begin{array}{l}\text { Bauhinia } \\
\text { racemosa Lam. }\end{array}$ & Fabaceae & Trees & $\begin{array}{l}\text { Hill forest, } \\
\text { bunds of } \\
\text { cultivated } \\
\text { fields }\end{array}$ & $\begin{array}{l}\text { Fruits, } \\
\text { Tender } \\
\text { leaves, } \\
\text { flowers }\end{array}$ & $\begin{array}{l}\text { Through } \\
\text { out year }\end{array}$ \\
\hline 18. & Pochi & $\begin{array}{l}\text { Blumea eriantha } \\
\text { DC. }\end{array}$ & Asteraceae & Herbs & $\begin{array}{l}\text { Wasteland } \\
\text { and open area }\end{array}$ & Leaves & Rainy \\
\hline 19. & Savari/ savar & Bombax ceiba $\mathrm{L}$. & Bombacaeae & Trees & Open forest & Flowers & Summer \\
\hline 20. & Asand & $\begin{array}{l}\text { Bridelia squamosa } \\
\text { (Lam.) Gehrm. }\end{array}$ & Euphorbiaceae & Small trees & $\begin{array}{l}\text { Forest } \\
\text { clearings }\end{array}$ & Fruits & Summer \\
\hline 21. & $\begin{array}{l}\text { Waghati / } \\
\text { Ghot }\end{array}$ & $\begin{array}{l}\text { Capparis } \\
\text { zeylanica } \mathrm{L} .\end{array}$ & Capparaceae & Climbers & $\begin{array}{l}\text { Dry scrub } \\
\text { forest }\end{array}$ & Fruits & Summer \\
\hline 22. & Karvand & $\begin{array}{l}\text { Carissa carandas } \\
\text { L. }\end{array}$ & Apocynaceae & $\begin{array}{l}\text { Thorny } \\
\text { shrubs }\end{array}$ & $\begin{array}{l}\text { Open hill } \\
\text { slopes }\end{array}$ & Fruits & Summer \\
\hline 23. & $\begin{array}{l}\text { Chothi } \\
\text { karvand }\end{array}$ & $\begin{array}{l}\text { Carrisa congesta } \\
\text { Wight }\end{array}$ & Apocynaceae & $\begin{array}{l}\text { Thorny } \\
\text { shrubs }\end{array}$ & $\begin{array}{l}\text { Open hill } \\
\text { slopes }\end{array}$ & Fruits & Summer \\
\hline 24. & Bahava & Cassia fistula $\mathrm{L}$. & Fabaceae & $\begin{array}{l}\text { Deciduous } \\
\text { trees }\end{array}$ & Forest area & $\begin{array}{l}\text { Tender } \\
\text { leaves, } \\
\text { flowers }\end{array}$ & $\begin{array}{l}\text { Rainy, } \\
\text { summer }\end{array}$ \\
\hline 25. & $\begin{array}{l}\text { Takala/ } \\
\text { Tarvata/ } \\
\text { Powadya }\end{array}$ & Cassia tora $\mathrm{L}$. & Fabaceae & Herbs & $\begin{array}{l}\text { Roadsides, } \\
\text { bunds of } \\
\text { cultivated } \\
\text { lands and } \\
\text { wasteland }\end{array}$ & $\begin{array}{l}\text { Tender } \\
\text { leaves }\end{array}$ & Rainy \\
\hline 26. & Gal/ Gela phal & $\begin{array}{l}\text { Catunaregam } \\
\text { spinosa (Thunb.) } \\
\text { Tirveng. }\end{array}$ & Rubiaceae & Tree & Open Forest & $\begin{array}{l}\text { Tender } \\
\text { fruits }\end{array}$ & Summer \\
\hline 27. & Kurdu & $\begin{array}{l}\text { Celosia argentea } \\
\text { L. var. argentea }\end{array}$ & Amaranthaceae & Herbs & $\begin{array}{l}\text { Wasteland and } \\
\text { Weed of } \\
\text { cultivated land }\end{array}$ & $\begin{array}{l}\text { Leaves, } \\
\text { tender stem }\end{array}$ & $\begin{array}{l}\text { Through } \\
\text { out year }\end{array}$ \\
\hline 28. & $\begin{array}{l}\text { Kavli Bhaji/ } \\
\text { Safed musali }\end{array}$ & $\begin{array}{l}\text { Chlorophytum } \\
\text { borivilianum } \\
\text { Santapau \& R.R. } \\
\text { Fern. }\end{array}$ & Asparagaceae & Herbs & Hilly slopes & $\begin{array}{l}\text { Tender } \\
\text { leaves }\end{array}$ & Rainy \\
\hline 29. & Kavala & $\begin{array}{l}\text { Chlorophytum } \\
\text { breviscapum Dalz. }\end{array}$ & Asparagaceae & Herbs & $\begin{array}{l}\text { Steep hill } \\
\text { slopes }\end{array}$ & $\begin{array}{l}\text { Tender } \\
\text { leaves }\end{array}$ & Rainy \\
\hline 30. & Savaliche pan & $\begin{array}{l}\text { Chlorophytum } \\
\text { nimmonii Dalzell }\end{array}$ & Asparagaceae & Herbs & $\begin{array}{l}\text { Rocky hill } \\
\text { slope in shady } \\
\text { places }\end{array}$ & Leaves & Rainy \\
\hline 31. & $\begin{array}{l}\text { Kavli Bhaji/ } \\
\text { Safed musali }\end{array}$ & $\begin{array}{l}\text { Chlorophytum } \\
\text { tuberosum } \\
\text { (Roxb.) Baker }\end{array}$ & Asparagaceae & Herbs & Hilly slopes & Leaves & Rainy \\
\hline 32. & $\begin{array}{l}\text { Alu, Teri, } \\
\text { Tera, Aohwlya }\end{array}$ & $\begin{array}{l}\text { Colocasia } \\
\text { esculenta (L.) } \\
\text { Schott. }\end{array}$ & Araceae & Herbs & $\begin{array}{l}\text { Shady, } \\
\text { marshy and } \\
\text { water logging } \\
\text { places }\end{array}$ & $\begin{array}{l}\text { Corm, } \\
\text { petiole, } \\
\text { Leaves }\end{array}$ & Rainy \\
\hline
\end{tabular}




\begin{tabular}{|c|c|c|c|c|c|c|c|}
\hline $\begin{array}{l}\text { Sl. } \\
\text { No. }\end{array}$ & $\begin{array}{l}\text { Common } \\
\text { name }\end{array}$ & Scientific name & Family & Habit & Habitat & Edible part & Season \\
\hline 33. & Keni & $\begin{array}{l}\text { Commelina } \\
\text { benghalensis L. }\end{array}$ & Commelinaceae & Herbs & $\begin{array}{l}\text { Open and } \\
\text { Wet places }\end{array}$ & Leaves & Rainy \\
\hline 34. & Chuch & $\begin{array}{l}\text { Corchorus } \\
\text { olitorius L. }\end{array}$ & Malvaceae & Herbs & $\begin{array}{l}\text { Wasteland, } \\
\text { weed of } \\
\text { cultivated } \\
\text { fields }\end{array}$ & $\begin{array}{l}\text { Tender } \\
\text { leaves, }\end{array}$ & Rainy \\
\hline 35. & $\begin{array}{l}\text { Bhokar/ Shelti } \\
\text { /Shelut }\end{array}$ & $\begin{array}{l}\text { Cordia dichotoma } \\
\text { G. Forst. }\end{array}$ & Boraginaceae & Trees & Forest areas & Fruits & Summer \\
\hline 36. & Pevkand & $\begin{array}{l}\text { Costus speciosus } \\
\text { (J. Koenig) Sm. }\end{array}$ & Costaceae & Herbs & $\begin{array}{l}\text { Forest } \\
\text { understory; } \\
\text { moist and } \\
\text { shady places }\end{array}$ & Flowers & Rainy \\
\hline 37. & Tag/ Tagada & $\begin{array}{l}\text { Crotalaria juncea } \\
\text { L. }\end{array}$ & Fabaceae & Herbs & $\begin{array}{l}\text { Wastelands, } \\
\text { roadside areas }\end{array}$ & Flowers & Winter \\
\hline 38. & Mek & $\begin{array}{l}\text { Cucumis setosus } \\
\text { Cogn. }\end{array}$ & Cucurbitaceae & Climbers & $\begin{array}{l}\text { Field bunds, } \\
\text { open forests }\end{array}$ & Fruits & Rainy \\
\hline 39. & $\begin{array}{l}\text { Bandgul/ } \\
\text { Bendgul/ } \\
\text { Bhedval }\end{array}$ & $\begin{array}{l}\text { Dendrophthoe } \\
\text { falcata L.f. }\end{array}$ & Loranthaceae & $\begin{array}{l}\text { Shrubs } \\
\text { (Stem } \\
\text { parasite) }\end{array}$ & $\begin{array}{l}\text { Parasite on } \\
\text { trees }\end{array}$ & fruit & summer \\
\hline 40. & $\begin{array}{l}\text { Konkand, } \\
\text { Konphal }\end{array}$ & $\begin{array}{l}\text { Dioscorea alata } \\
\text { L. }\end{array}$ & Dioscoreaceae & Climbers & $\begin{array}{l}\text { Cultivated but } \\
\text { many places } \\
\text { naturalized }\end{array}$ & $\begin{array}{l}\text { Tuber, } \\
\text { bulbil }\end{array}$ & Rainy \\
\hline 41. & $\begin{array}{l}\text { Kadu karanda, } \\
\text { Kadu kand }\end{array}$ & $\begin{array}{l}\text { Dioscorea } \\
\text { bulbifera } \mathrm{L} .\end{array}$ & Dioscoreaceae & Climbers & $\begin{array}{l}\text { Forest borders } \\
\text { on hedges }\end{array}$ & $\begin{array}{l}\text { Tuber, } \\
\text { bulbil }\end{array}$ & Rainy \\
\hline 42. & Ulshi & $\begin{array}{l}\text { Dioscorea hispida } \\
\text { Dennst. }\end{array}$ & Dioscoreaceae & Climbers & $\begin{array}{l}\text { On hedges } \\
\text { and bushes }\end{array}$ & $\begin{array}{l}\text { Tuber, } \\
\text { bulbil }\end{array}$ & Rainy \\
\hline 43. & Chai vel & $\begin{array}{l}\text { Dioscorea } \\
\text { pentaphylla } \mathrm{L} .\end{array}$ & Dioscoreaceae & Climbers & $\begin{array}{l}\text { Forest borders } \\
\text { on hedges }\end{array}$ & $\begin{array}{l}\text { Inflorescen } \\
\text { ce }\end{array}$ & Rainy \\
\hline 44. & Tembhurni & $\begin{array}{l}\text { Diospyros } \\
\text { melanoxylon } \\
\text { Roxb. }\end{array}$ & Ebenaceae & Small trees & $\begin{array}{l}\text { Deciduous } \\
\text { forest }\end{array}$ & $\begin{array}{l}\text { Unripe and } \\
\text { ripe Fruits }\end{array}$ & $\begin{array}{l}\text { Winter/ } \\
\text { summer }\end{array}$ \\
\hline 45. & Awala & $\begin{array}{l}\text { Emblica } \\
\text { officinalis Gaertn. }\end{array}$ & Euphorbiaceae & Small trees & $\begin{array}{l}\text { Open forest } \\
\text { and cultivated }\end{array}$ & Fruits & Winter \\
\hline 46. & $\begin{array}{l}\text { Kelful } \\
\text { /Bankel/ } \\
\text { Rankel/ } \\
\text { Kavadar }\end{array}$ & $\begin{array}{l}\text { Ensete superbum } \\
\text { (Roxb.) Cheesm. }\end{array}$ & Musaeae & Herbs & $\begin{array}{l}\text { Steep rocky } \\
\text { hill slopes }\end{array}$ & $\begin{array}{l}\text { Rhizome, } \\
\text { young } \\
\text { shoot, } \\
\text { flowers } \\
\text { Immature } \\
\text { fruit }\end{array}$ & Rainy \\
\hline 47. & Umbar & Ficus racemosa $\mathrm{L}$. & Moraceae & Trees & $\begin{array}{l}\text { Forest areas, } \\
\text { along streams } \\
\text { and cultivated }\end{array}$ & Fruit & Summer \\
\hline 48. & Ghugurval & $\begin{array}{l}\text { Flacourtia indica } \\
\text { (Burm. f.) Merr. }\end{array}$ & Salicaceae & Trees & $\begin{array}{l}\text { Dry } \\
\text { deciduous } \\
\text { forests }\end{array}$ & fruits & Summer \\
\hline 49. & Kakad & $\begin{array}{l}\text { Garuga pinnata } \\
\text { Roxb. }\end{array}$ & Burseraceae & Trees & Hill forest & Fruit & Summer \\
\hline 50. & Dhaman & $\begin{array}{l}\text { Grewia tiliifolia } \\
\text { Vahl. }\end{array}$ & Malvaceae & Trees & Forest area & fruit & $\begin{array}{l}\text { Summer, } \\
\text { winter }\end{array}$ \\
\hline 51. & $\begin{array}{l}\text { Khadak } \\
\text { ambadi }\end{array}$ & $\begin{array}{l}\text { Hibiscus caesius } \\
\text { Garcke. }\end{array}$ & Malvaceae & Shrubs & $\begin{array}{l}\text { Weed on road } \\
\text { side }\end{array}$ & fruit, leaves & Rainy \\
\hline
\end{tabular}




\begin{tabular}{|c|c|c|c|c|c|c|c|}
\hline $\begin{array}{l}\text { Sl. } \\
\text { No. }\end{array}$ & $\begin{array}{l}\text { Common } \\
\text { name }\end{array}$ & Scientific name & Family & Habit & Habitat & Edible part & Season \\
\hline 52. & Dumkha & $\begin{array}{l}\text { Hibiscus } \\
\text { cannabinus L. }\end{array}$ & Malvaceae & Herbs & $\begin{array}{l}\text { Mostly } \\
\text { cultivated } \\
\text { sometimes } \\
\text { escaped }\end{array}$ & $\begin{array}{l}\text { Leaves, } \\
\text { tender } \\
\text { shoots, } \\
\text { flower }\end{array}$ & $\begin{array}{l}\text { Rainy, } \\
\text { stored } \\
\text { for lean } \\
\text { season }\end{array}$ \\
\hline 53. & Dumkha & $\begin{array}{l}\text { Hibiscus } \\
\text { sabdariffa } \mathrm{L} .\end{array}$ & Malvaceae & Herbs & $\begin{array}{l}\text { Mostly } \\
\text { cultivated } \\
\text { sometimes } \\
\text { escaped }\end{array}$ & $\begin{array}{l}\text { Leaves, } \\
\text { tender } \\
\text { shoots, } \\
\text { flower }\end{array}$ & $\begin{array}{l}\text { Rainy, } \\
\text { stored } \\
\text { for lean } \\
\text { season }\end{array}$ \\
\hline 54. & Kuda & $\begin{array}{l}\text { Holarrhena } \\
\text { pubescens Wall. } \\
\text { ex G. Don }\end{array}$ & Apocynaceae & Trees & Hill slopes & Flowers & Summer \\
\hline 55. & Shiri & $\begin{array}{l}\text { Holostemma ada- } \\
\text { kodien Schult. }\end{array}$ & Apocynaceae & Climbers & Forest area & $\begin{array}{l}\text { Flower, } \\
\text { Fruit }\end{array}$ & Summer \\
\hline 56. & Bondara & $\begin{array}{l}\text { Lagerstroemia } \\
\text { parviflora } \text { Roxb. }\end{array}$ & Lythraceae & Trees & Hill slopes & Leaves & $\begin{array}{l}\text { Rainy, } \\
\text { summer }\end{array}$ \\
\hline 57. & Pathari & $\begin{array}{l}\text { Launaea } \\
\text { intybacea (Jacq.) } \\
\text { Beauverd }\end{array}$ & Asteraceae & Herbs & $\begin{array}{l}\text { Weed of } \\
\text { cultivated } \\
\text { fields and } \\
\text { wastelands }\end{array}$ & Leaves & $\begin{array}{l}\text { Rainy, } \\
\text { winter }\end{array}$ \\
\hline 58. & Akkarghode & $\begin{array}{l}\text { Launaea } \\
\text { procumbens } \\
\text { (Roxb.) Ramayya } \\
\text { \& Rajgopal. }\end{array}$ & Asteraceae & Herbs & $\begin{array}{l}\text { Weed of } \\
\text { cultivated } \\
\text { fields and } \\
\text { wastelands }\end{array}$ & Leaves & $\begin{array}{l}\text { Rainy, } \\
\text { winter }\end{array}$ \\
\hline 59. & Motha dinda & $\begin{array}{l}\text { Leea macrophylla } \\
\text { Roxb. Ex } \\
\text { Hornem. }\end{array}$ & Vitaceae & Shrubs & Hill forest & $\begin{array}{l}\text { Young } \\
\text { leaves }\end{array}$ & Rainy \\
\hline 60. & Mahua & $\begin{array}{l}\text { Madhuca } \\
\text { longifolia (J. } \\
\text { Koenig ex L.) J.F. } \\
\text { Macbr. }\end{array}$ & Sapotaceae & Trees & $\begin{array}{l}\text { Forest area, } \\
\text { bunds of } \\
\text { cultivated } \\
\text { lands }\end{array}$ & $\begin{array}{l}\text { Flower, } \\
\text { fruit, seeds }\end{array}$ & Summer \\
\hline 61. & $\begin{array}{l}\text { Ambat } \\
\text { Bintukli }\end{array}$ & $\begin{array}{l}\text { Maesa indica } \\
\text { (Roxb.) A. DC. }\end{array}$ & Myrsinaceae & Shrubs & $\begin{array}{l}\text { Forest } \\
\text { clearings }\end{array}$ & Leaves & $\begin{array}{l}\text { Through } \\
\text { out year }\end{array}$ \\
\hline 62. & $\begin{array}{l}\text { Aliv, Helu, } \\
\text { Alu }\end{array}$ & $\begin{array}{l}\text { Meyna laxiflora } \\
\text { Robyns }\end{array}$ & Rubiaceae & $\begin{array}{l}\text { Thorny } \\
\text { small trees }\end{array}$ & $\begin{array}{l}\text { Moist } \\
\text { deciduous } \\
\text { forest }\end{array}$ & Fruits & $\begin{array}{l}\text { Late } \\
\text { Summer }\end{array}$ \\
\hline 63. & Humb & $\begin{array}{l}\text { Miliusa tomentosa } \\
\text { (Roxb.) J. Sinclair }\end{array}$ & Annonaceae & Trees & Open forest & fruits & Summer \\
\hline 64. & Kartoli & $\begin{array}{l}\text { Momordica dioica } \\
\text { Roxb.ex. Wild }\end{array}$ & Cucurbitaceae & Climbers & $\begin{array}{l}\text { Open steep } \\
\text { hill slope }\end{array}$ & Fruits & Rainy \\
\hline 65. & $\begin{array}{l}\text { Kadu shevaga/ } \\
\text { Ran shevga }\end{array}$ & $\begin{array}{l}\text { Moringa } \\
\text { concanensis } \\
\text { Nimmo }\end{array}$ & Moringaceae & Trees & Forest area & $\begin{array}{l}\text { Leaves, } \\
\text { flowers, } \\
\text { fruits }\end{array}$ & $\begin{array}{l}\text { Through } \\
\text { out year }\end{array}$ \\
\hline 66. & Shevga & $\begin{array}{l}\text { Moringa oleifera } \\
\text { Lam. }\end{array}$ & Moringaceae & Trees & $\begin{array}{l}\text { Community } \\
\text { land, } \\
\text { cultivated on } \\
\text { back yard }\end{array}$ & $\begin{array}{l}\text { Leaves, } \\
\text { flowers, } \\
\text { fruits }\end{array}$ & $\begin{array}{l}\text { Through } \\
\text { out year }\end{array}$ \\
\hline 67. & Tetu & $\begin{array}{l}\text { Oroxylum indicum } \\
\text { (L.) Kurz }\end{array}$ & Bignoniaceae & Trees & Open forest & $\begin{array}{l}\text { Young } \\
\text { fruits }\end{array}$ & Rainy \\
\hline 68. & Ladukli & $\begin{array}{l}\text { Pavetta tomentosa } \\
\text { Roxb ex Sm. }\end{array}$ & Rubiaceae & Shrubs & $\begin{array}{l}\text { Forest } \\
\text { understory }\end{array}$ & Flowers & Winter \\
\hline 69. & Bhafali & $\begin{array}{l}\text { Pimpinella } \\
\text { wallichiana } \\
\text { Gandhi. }\end{array}$ & Apiaceae & Herbs & $\begin{array}{l}\text { Grassland } \\
\text { associate }\end{array}$ & Leaves & Rainy \\
\hline
\end{tabular}




\begin{tabular}{|c|c|c|c|c|c|c|c|}
\hline $\begin{array}{l}\text { Sl. } \\
\text { No. }\end{array}$ & $\begin{array}{l}\text { Common } \\
\text { name }\end{array}$ & Scientific name & Family & Habit & Habitat & Edible part & Season \\
\hline 70. & Ghol & $\begin{array}{l}\text { Portulaca } \\
\text { oleracea L. }\end{array}$ & Portulacaceae & Herbs & $\begin{array}{l}\text { Weed of } \\
\text { cultivated and } \\
\text { waste land }\end{array}$ & $\begin{array}{l}\text { Tender } \\
\text { stem and } \\
\text { leaves }\end{array}$ & Rainy \\
\hline 71. & Kharshenga & $\begin{array}{l}\text { Radermachera } \\
\text { xylocarpa (Roxb.) } \\
\text { Roxb. ex K. } \\
\text { Schum. }\end{array}$ & Bignoniaceae & Trees & $\begin{array}{l}\text { Deciduous } \\
\text { forest }\end{array}$ & $\begin{array}{l}\text { Young } \\
\text { fruits }\end{array}$ & Winter \\
\hline 72. & Lothi & $\begin{array}{l}\text { Sauromatum } \\
\text { venosum (Aiton) } \\
\text { Kunth }\end{array}$ & Araceae & Herbs & $\begin{array}{l}\text { Forest } \\
\text { understory in } \\
\text { rock crevices }\end{array}$ & $\begin{array}{l}\text { Ripe fruits, } \\
\text { leaves }\end{array}$ & Summer \\
\hline 73. & Koshim & $\begin{array}{l}\text { Schleichera } \\
\text { oleosa } \\
\text { (Lour.)Oken }\end{array}$ & Sapindaceae & Trees & Forest borders & Ripe fruits & summer \\
\hline 74. & Mokha & $\begin{array}{l}\text { Schrebera } \\
\text { swietenioides } \\
\text { Roxb. }\end{array}$ & Oleaceae & $\begin{array}{l}\text { Deciduous } \\
\text { trees }\end{array}$ & Forest area & $\begin{array}{l}\text { Tender } \\
\text { Leaves }\end{array}$ & $\begin{array}{l}\text { Winter, } \\
\text { summer }\end{array}$ \\
\hline 75. & Hadaga & $\begin{array}{l}\text { Sesbania } \\
\text { grandiflora L. } \\
\text { Poir. }\end{array}$ & Fabaceae & Trees & $\begin{array}{l}\text { Cultivated in } \\
\text { kitchen } \\
\text { garden but } \\
\text { naturalized in } \\
\text { many places }\end{array}$ & Flowers & Rainy \\
\hline 76. & Kuala & $\begin{array}{l}\text { Smithia conferta } \\
\text { Sm. }\end{array}$ & Fabaceae & Herbs & $\begin{array}{l}\text { Grassland } \\
\text { associate }\end{array}$ & $\begin{array}{l}\text { Tender } \\
\text { Leaves }\end{array}$ & Rainy \\
\hline 77. & Kavali bhaji & $\begin{array}{l}\text { Smithia hirsuta } \\
\text { Dalzell. }\end{array}$ & Fabaceae & Herbs & $\begin{array}{l}\text { Grassland } \\
\text { associate }\end{array}$ & $\begin{array}{l}\text { Tender } \\
\text { Leaves }\end{array}$ & Rainy \\
\hline 78. & Donger Bhaji & $\begin{array}{l}\text { Solena } \\
\text { amplexicaulis } \\
\text { (Lam.) Gandhi }\end{array}$ & Cucurbitaceae & Climbers & $\begin{array}{l}\text { Hill slopes on } \\
\text { bushes }\end{array}$ & Fruits & $\begin{array}{l}\text { Rainy, } \\
\text { winter }\end{array}$ \\
\hline 79. & Khadak teri & $\begin{array}{l}\text { Sonerila } \\
\text { scapigera Dalzell }\end{array}$ & Melastomataceae & Herbs & $\begin{array}{l}\text { Vertical rocks } \\
\text { and tree } \\
\text { trunks }\end{array}$ & Leaves & Rainy \\
\hline 80. & Borthada & $\begin{array}{l}\text { Sphaeranthus } \\
\text { indicus } \mathrm{L} \text {. }\end{array}$ & Asteraceae & Herbs & Wastelands & $\begin{array}{l}\text { Tender } \\
\text { Leaves }\end{array}$ & Rainy \\
\hline 81. & Ambada & $\begin{array}{l}\text { Spondias pinnata } \\
\text { (L. f.) Kurz }\end{array}$ & Anacardiaceae & Trees & Forest area & $\begin{array}{l}\text { Young } \\
\text { leaves, } \\
\text { fruits }\end{array}$ & Summer \\
\hline 82. & Kukurval & $\begin{array}{l}\text { Sterculia guttata } \\
\text { Roxb. }\end{array}$ & Sterculiaceae & Trees & $\begin{array}{l}\text { Deciduous } \\
\text { forest }\end{array}$ & Seeds & Summer \\
\hline 83. & Kahandol & $\begin{array}{l}\text { Sterculia urens } \\
\text { Roxb. }\end{array}$ & Sterculiaceae & Trees & $\begin{array}{l}\text { Deciduous } \\
\text { forest }\end{array}$ & Seeds & Summer \\
\hline 84. & Chinch & $\begin{array}{l}\text { Tamarindus } \\
\text { indica } \mathrm{L} .\end{array}$ & Fabaceae & Trees & $\begin{array}{l}\text { Social } \\
\text { forestry, road } \\
\text { side } \\
\text { cultivation }\end{array}$ & $\begin{array}{l}\text { Tender } \\
\text { leaves } \\
\text { Flowers, } \\
\text { fruits, seeds }\end{array}$ & Winter \\
\hline 85. & Pendhara & $\begin{array}{l}\text { Tamilnalia } \\
\text { uliginosa (Retz.) } \\
\text { Tirveng \& Sastre }\end{array}$ & Rubiaceae & Trees & Scrub forest & Fruits & Rainy \\
\hline 86. & Garbhend & $\begin{array}{l}\text { Thespesia } \\
\text { populnea (L.) } \\
\text { Soland ex. Corr. }\end{array}$ & Malvaceae & Trees & $\begin{array}{l}\text { Social } \\
\text { forestry, road } \\
\text { side } \\
\text { cultivation }\end{array}$ & $\begin{array}{l}\text { Tender } \\
\text { Leaves }\end{array}$ & $\begin{array}{l}\text { Through } \\
\text { out year }\end{array}$ \\
\hline 87. & Ran udid & Vigna radiata & Fabaceae & Herbs & $\begin{array}{l}\text { Grassland } \\
\text { associate }\end{array}$ & $\begin{array}{l}\text { Tender } \\
\text { Leaves, } \\
\text { seeds }\end{array}$ & Rainy \\
\hline
\end{tabular}




\begin{tabular}{|c|c|c|c|c|c|c|c|}
\hline $\begin{array}{l}\text { SI. } \\
\text { No. }\end{array}$ & $\begin{array}{l}\text { Common } \\
\text { name }\end{array}$ & Scientific name & Family & Habit & Habitat & Edible part & Season \\
\hline 88. & Dudh halinda & $\begin{array}{l}\text { Vigna vexillata } \\
\text { (L.) A. Rich. }\end{array}$ & Fabaceae & Climbers & Forest borders & $\begin{array}{l}\text { Tuber, } \\
\text { seeds }\end{array}$ & Rainy \\
\hline 89. & Kali kudai & $\begin{array}{l}\text { Wrightia tinctoria } \\
\text { R.Br. }\end{array}$ & Apocynaceae & Trees & Forest & Flowers & Summer \\
\hline 90. & Bor & $\begin{array}{l}\text { Ziziphus } \\
\text { mauritiana Lam. }\end{array}$ & Rhamnaceae & Trees & $\begin{array}{l}\text { Open forest, } \\
\text { bunds }\end{array}$ & Fruits & Summer \\
\hline 91. & Toran & $\begin{array}{l}\text { Ziziphus rugosa } \\
\text { Lam. }\end{array}$ & Rhamnaceae & $\begin{array}{l}\text { Woody } \\
\text { climbers }\end{array}$ & $\begin{array}{l}\text { Forest } \\
\text { margins }\end{array}$ & Fruits & Summer \\
\hline
\end{tabular}

